\title{
Impact of Mobile Sink for Wireless Sensor Networks Considering Different Radio Models and Performance Metrics
}

\author{
Tao Yang*, Gjergji Mino ${ }^{\dagger}$, Leonard Barolli ${ }^{\ddagger}$, Fatos Xhafa ${ }^{\S}$ and Arjan Durresi $\uparrow$ \\ * JSPS Fellow Researcher \\ Fukuoka Institute of Technology (FIT) \\ 3-30-1 Wajiro-Higashi, Higashi-Ku, Fukuoka 811-0295, Japan \\ Email: taoyang.tou@gmail.com \\ $\dagger$ Graduate School of Engineering \\ Fukuoka Institute of Technology (FIT) \\ 3-30-1 Wajiro-Higashi, Higashi-Ku, Fukuoka 811-0295, Japan \\ Email: bd09002@bene.fit.ac.jp \\ ${ }^{\ddagger}$ Department of Information and Communication Engineering \\ Fukuoka Institute of Technology (FIT) \\ 3-30-1 Wajiro-Higashi, Higashi-Ku, Fukuoka 811-0295, Japan \\ E-mail: barolli@fit.ac.jp \\ $\S$ Technical University of Catalonia \\ Department of Languages and Informatics Systems \\ C/Jordi Girona 1-3, 08034 Barcelona, Spain \\ E-mail:fatos@lsi.upc.edu \\ IDepartment of Computer and Information Science \\ Indiana University Purdue University at Indianapolis (IUPUI) \\ 723 W. Michigan Street SL 280 Indianapolis, IN 46202, USA \\ E-mail:durresi@cs.iupui.edu
}

\begin{abstract}
In this work, we investigate how WSN performs in the case when sink node moves using different radio models and metrics. We consider routing efficiency, delay and number of received packet metrics to evaluate the performance of WSN using AODV routing protocol, lattice topology, and TwoRayGround and Shadowing radio models. We evaluate the performance of WSN by simulations. The performance evaluation results show that for small number of sensor nodes, the RE of Shadowing radio model is better than TwoRayGround model. However, for high node density, the RE of TwoRayGround model is better than Shadowing radio model. When $T_{r}$ is less 10 , the delay of Shadowing model is better than TwoRayGround model. However, for $T_{r}$ larger than 100, the delay of TwoRayGround model is better than Shadowing model. With increase of the number of nodes, the number of received packets is also increased. For TwoRayGround model, when $T_{r}$ is less than 10 , the number of received packets for 16, 64, and 100 nodes is almost the same. However, comparing TwoRayGround model with Shadowing model, for the same time interval and for the same number of nodes, the number of received packets of TwoRayGround model is better than Shadowing model.
\end{abstract}

Keywords-Sensor network, Mobile sink, Topology, Routing Efficiency, Delay, Received Packet.

\section{INTRODUCTION}

A Wireless Sensor Networks (WSNs) consists of spatially distributed autonomous sensors to cooperatively monitor physical or environmental conditions, such as temperature, sound, vibration, pressure, motion or pollutants. The development of WSNs was motivated by military applications such as battlefield surveillance. They are now used in many industrial and civilian application areas, including industrial process monitoring and control, machine health monitoring, environment and habitat monitoring, healthcare applications, home automation, and traffic control.

Each node in a WSN is typically equipped with a radio transceiver or other wireless communication devices, a small micro-controller, and an energy source (usually a battery). A sensor node might vary in size from that of a shoebox down to the size of a grain of dust, although functioning "motes" of genuine microscopic dimensions have yet to be created. The cost of sensor nodes is similarly variable, ranging from hundreds of dollars to a few pennies, depending on the size of the WSNs and the complexity required of individual sensor nodes. Size and cost constraints on sensor nodes 
result in corresponding constraints on resources such as energy, memory, computational speed and bandwidth.

There are many applications of WSNs. For instance, in military application, the rapid deployment, self-organization, and fault-tolerance characteristics of sensor nodes make them a promising, surveillance, reconnaissance, and targeting systems. In health car, sensor nodes can be used to monitor patients and assist disabled patients. Other applications include managing inventory, monitoring product quality, and monitoring disaster areas.

Recently, there are many research work for WSNs[1], [2], [3], [4]. In this paper, we study a particular application of WSN for event-detection and tracking. The application is based on the assumption that WSN present some degree of spatial redundancy. For instance, whenever an event happens, a certain event data is transmitted to the sink node. Because of the spatial redundancy, we can tolerate some packet loss, as long as the required detection or eventreliability holds. This reliability can be formulated as the minimum number of packets required by the sink node in order to re-construct the event field. We want to investigate the performance of WSN for different topologies considering a single mobile sink. In the large scale network, the sink node is faraway from the sensor nodes. For this reason, it is needed more energy to send the sensed data. By using mobile sink, the consumed energy of sensor nodes can be reduced.

The remainder of the paper is organized as follows. In Section II, we present the related work. In Section III, we explain the proposed network simulation model. In Section $\mathrm{IV}$, we discuss the RE, delay and number of received packets. In Section V, we show the simulation results. Conclusions of the paper are given in Section VI.

\section{RELATED WORK}

In our previous work [5], we implemented a simulation system for WSNs considering different protocols and different propagation radio models. We did not consider the sink movement. The authors of [6] suggest a reinforcement learning algorithm for sensor nodes that they call Hybrid Learning-Enforced Time Domain Routing (HLETDR). Each node continuously learns the movement pattern of the mobile sink and statistically characterize it as a probability distribution function. Thus, sensor nodes always know in which direction they have to route messages to the sink at a given time instant. The advantage of the solution is that nodes do not need time synchronization, since they make forwarding decisions in their local time-domain.

In [7], the authors consider scenarios where sensors are deployed within a circle. The authors argue that in such cases the mobile sink should follow the periphery of the network in order to optimize the energy consumption of the nodes.

In our previous work [8], we obtained the simulation results for consumed energy in case of mobile sink. We

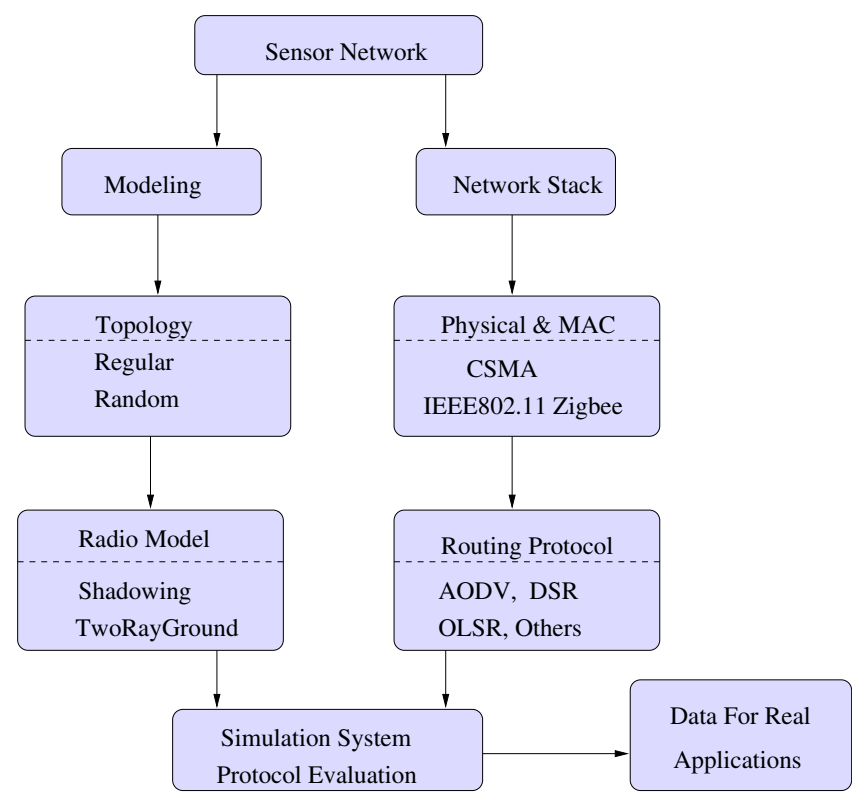

Figure 1. Network simulation model.

found that the consumed energy of mobile sink is better than the stationary sink (about half of stationary in lattice topology). The goodput of random topology is better than lattice topology and the consumed energy of lattice topology is better than random topology.

However, the related work deal with specific problems of WSNs such WSN topology or propagation radio model. In our work, we propose and implement a trade-off method that considers different radio models, different routing protocols, different topologies, different MAC protocols and different sensor node density.

In this work, we assume a network consisting of 16 , 64, 100 sensor nodes and one mobile sink, which moves continuously on an arbitrary unknown path. The position of the sink cannot be determined in advance. Sensor nodes have limited radio range, thus multi-hop communication is used in the network. We consider Shadowing and TwoRayGround radio models and use AODV routing protocol and lattice topology for performance evaluation.

\section{Proposed Network Simulation Model}

In our WSN, every node detects the physical phenomenon and sends back to the sink node the data packets. We suppose that the sink node is more powerful than sensor nodes. In our previous work, the sink node was stationary. In this work, we consider that the sink is mobile. We analyse the performance of the network in a fixed time interval, which is the available time for the detection of the phenomenon and its value is application dependent.

Proposed network simulation model is shown in Fig. 1. For simulation system implementation, we consider mod- 


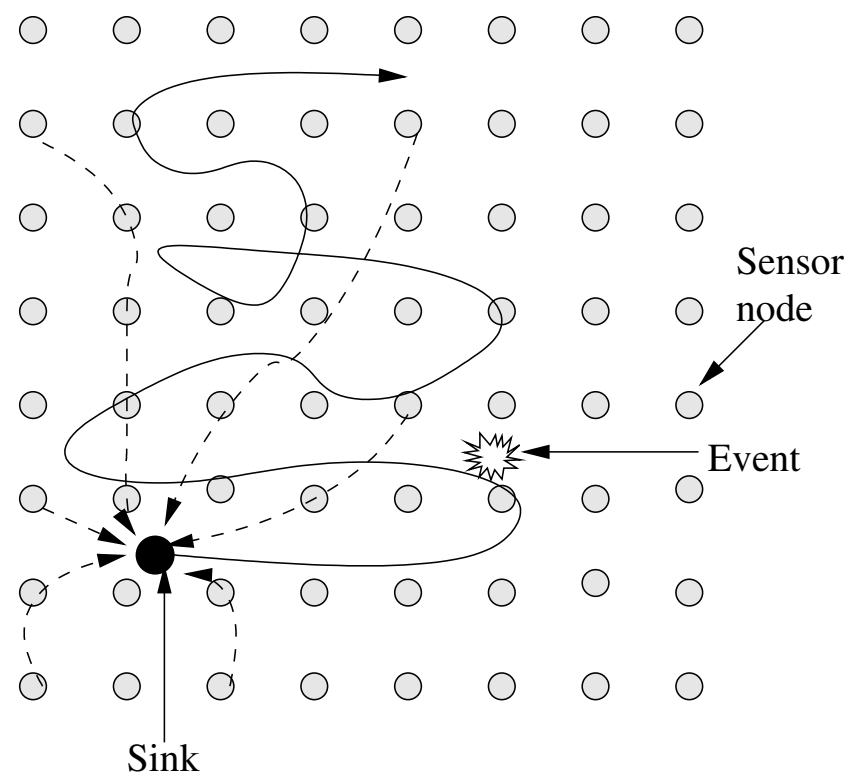

Figure 2. One pattern of mobile sink path.

elling and network stack. In this paper, we consider that a mobile sink is moving randomly in the WSN field. In Fig. 2 is shown one pattern of mobile sink path. We evaluated the $\mathrm{RE}$, delay and number of received packets of AODV protocol using TwoRayGround and Shadowing radio models for the lattice topology.

\section{A. Topology}

For the physical layout of the WSN, two types of deployment has been studied so far: the random and the lattice deployment. In the former, nodes are supposed to be uniformly distributed, while in the latter one nodes are vertexes of particular geometric shape, e.g. a square grid, as depicted in Fig. 3. For space constraints, here we present only the results for the lattice topology. In this case, in order to guarantee the connectedness of the network we should set the transmission range of every node to the step size, $d$, which is the minimum distance between two rows (or columns) of the grid. In fact, by this way the number of links that every node can establish (the node degree D) is 4. Nodes at the borders have $D=2$.

\section{B. Radio Models}

In order to simulate the detection of a natural event, we used the libraries from Naval Research Laboratory (NRL) [9]. In this framework, a phenomenon is modelled as a wireless mobile node. The phenomenon node broadcasts packets with a tunable synchrony or pulse rate, which represents the period of occurrence of a generic event.

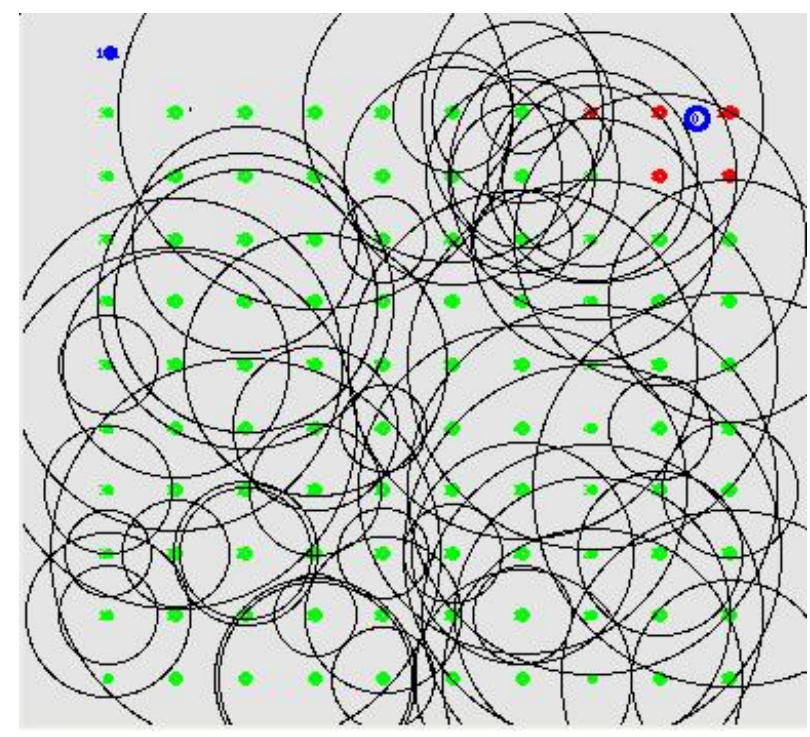

Figure 3. An example of lattice network.

These libraries provide the sensor node with an alarm variable. The alarm variable is a timer variable. It turns off the sensor if no event is sensed within an alarm interval. In addition to the sensing capabilities, every sensor can establish a multi-hop communication towards the Monitoring Node (MN) by means of a particular routing protocol.

We assume that the MAC protocol is the IEEE 802.11 standard. This serves to us as a baseline of comparison for other contention resolution protocols. The receiver of every sensor node is supposed to receive correctly data bits if the received power exceeds the receiver threshold, $\gamma$. This threshold depends on the hardware. It should be noted that other MAC factors affect the reception process, for example the Carrier Sensing Threshold (CST) and Capture Threshold (CP) of IEEE.802.11 used in Ns-2.

As reference, we select parameters values according to the features of a commercial device (MICA2 OEM). In particular, for this device, we found that for a carrier frequency of $f=916 \mathrm{MHz}$ and a data rate of $34 \mathrm{KBaud}$, we have a threshold (or receiver sensitivity) $\left.\gamma\right|_{d B}=-118 \mathrm{dBm}$ [10].

In Fig. 4 and Fig. 5 is shown the transmission range of TwoRayGround and Shadowing models. In particular, the emitted power of the phenomenon is calculated according to a TwoRayGround propagation model [11]. The Shadowing model assumes that the received power at the sensor node is: 


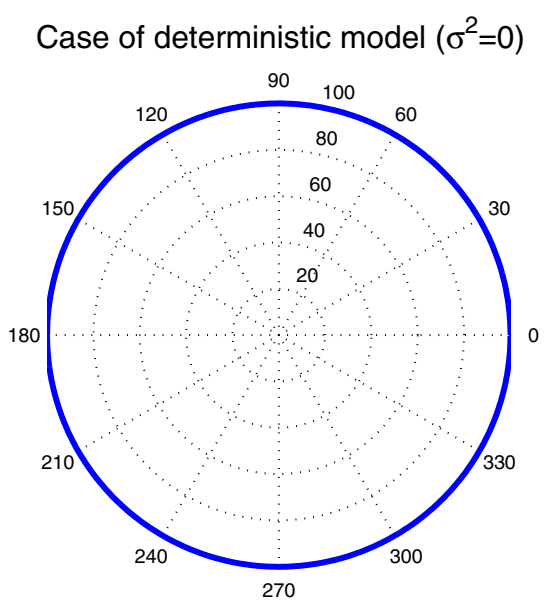

Figure 4. Transmission range of TwoRayGround model.

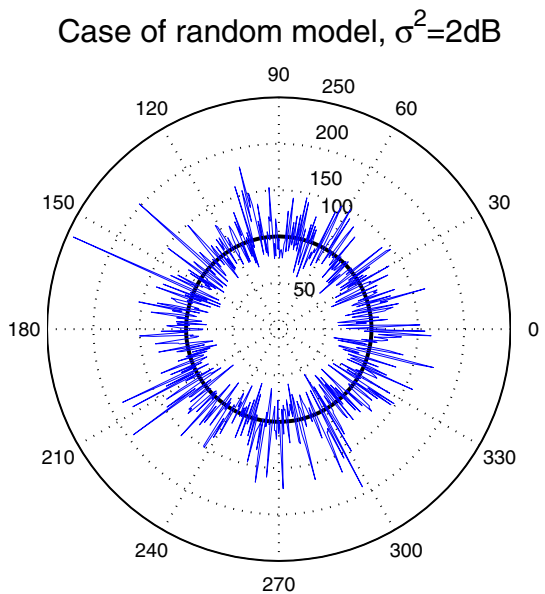

Figure 5. Transmission range of Shadowing model.

$$
\left.P_{r}(d)\right|_{\mathrm{dB}}=\underbrace{\left.P_{t}\right|_{\mathrm{dB}}-\beta_{0}-10 \alpha \log \left(\frac{d}{d_{0}}\right)}_{\text {deterministic part }}+\underbrace{S_{\mathrm{dB}}}_{\text {random part }}
$$

where $\beta_{0}$ is a constant. The term $S_{\mathrm{dB}}$ is a random variable, which accounts for random variations of the path loss. This variable is also known as log-normal Shadowing, because it is supposed to be Gaussian distributed with zero mean and variance $\sigma_{\mathrm{dB}}^{2}$, that is $S_{\mathrm{dB}} \sim \mathcal{N}\left(0, \sigma_{\mathrm{dB}}^{2}\right)$.

Given two nodes, if $P_{r}>\gamma$, where $\gamma$ is the hardware- dependent threshold, the link can be established. The case of $\sigma=0, \alpha=4, d>d_{0}$ is also called the TwoRaysGround model and it is a deterministic model, where in addition to the direct ray from the transmitter towards the receiver node, a ground reflected signal is supposed to be present. Accordingly, the received power now depends also on the antenna heights and the pathloss is:

$$
\beta=10 \log \left(\frac{(4 \pi d)^{4} L}{G_{t} G_{r} h_{t} h_{r} \lambda^{2}}\right)
$$

where $h_{r}$ and $h_{t}$ are the receiver and transmitter antenna heights, respectively.

\section{Energy Model}

The energy model concerns the dynamics of energy consumption of the sensor. A widely used model is as follows [12]. When the sensor transmits $k$ bits, the radio circuitry consumes an energy of $k P_{T x} T_{B}$, where $P_{T x}$ is the power required to transmit a bit which lasts $T_{B}$ seconds. By adding the radiated power $P_{t}(d)$, we have:

$$
E_{T x}(k, d)=k T_{B}\left(P_{T x}+P_{t}(d)\right) .
$$

Since packet reception consumes energy, by following the same reasoning, we have:

$$
E(k, d)=k P_{T x} T_{B}+k T_{B} P_{t}(d)+k P_{R x} T_{B}
$$

where $P_{R x}$ is the power required to correctly receive (demodulate and decode) one bit.

\section{Interference}

In general, in every wireless network the electromagnetic interference of neighbouring nodes is always present. The interference power decreases the Signal-to-Noise-Ratio (SNR) at the intended receiver, which will perceive a lower bit and/or packet error probability. Given a particular node, the interference power depends on how many transmitters are transmitting at the same time of the transmission of the given node. In a WSN, since the number of concurrent transmissions is low because of the low duty-cycle of sensors, we can neglect the interference. In other words, if we define duty-cycle as the fraction between the total time of all transmissions of sensor data and the total operational time of the network, we get always a value less than 0.5. In fact, the load of each sensor is $\ll 1$ because sensors transmit data only when an event is detected [12]. However, it is intuitive that in a more realistic scenario, where many phenomena trigger many events, the traffic load can be higher, and then the interference will worsen the performance.

\section{E. Routing Protocol}

We are aware of many proposals of routing protocols for ad-hoc networks [13]. Here, we consider AODV protocol. The AODV is an improvement of DSDV to on-demand scheme. It minimize the broadcast packet by creating route 
only when needed. Every node in network maintains the route information table and participate in routing table exchange. When source node wants to send data to the destination node, it first initiates route discovery process. In this process, source node broadcasts Route Request (RREQ) packet to its neighbours. Neighbour nodes which receive RREQ forward the packet to its neighbour nodes. This process continues until RREQ reach to the destination or the node who knows the path to destination. When the intermediate nodes receive RREQ, they record in their tables the address of neighbours, thereby establishing a reverse path. When the node which knows the path to destination or destination node itself receive RREQ, it send back Route Reply (RREP) packet to source node. This RREP packet is transmitted by using reverse path. When the source node receives RREP packet, it can know the path to destination node and it stores the discovered path information in its route table. This is the end of route discovery process. Then, AODV performs route maintenance process. In route maintenance process, each node periodically transmits Hello messages to detect link breakage.

\section{F. Event Detection and Transport}

We use the data-centric model similar to [14], where the end-to-end reliability is transformed into a bounded signal distortion concept. In this model, after sensing an event, every sensor node sends sensed data towards the MN. The transport used is a UDP-like transport, i.e. there is not any guarantee on the data delivery. While this approach reduces the complexity of the transport protocol and well fit the energy and computational constraints of sensor nodes, the event-reliability can be guaranteed to some extent because of the spatial redundancy. The sensor node transmits data packets reporting the details of the detected event at a certain transmission rate. The setting of this parameter, $T_{r}$, depends on several factors, as the quantization step of sensors, the type of phenomenon, and the desired level of distortion perceived at the MN. In [15], the authors used this $T_{r}$ as a control parameter of the overall system. For example, if we refer to event-reliability as the minimum number of packets required at sink in order to reliably detect the event, then whenever the sink receives a number of packets less than the event-reliability, it can instruct sensor nodes to use a higher $T_{r}$. This instruction is piggy-backed in dedicated packets from the MN. This system can be considered as a control system, as shown in Fig. 6, with the target event-reliability as input variable and the actual event-reliability as output parameter. The target event-reliability is transformed into an initial $T_{r}^{0}$. The control loop has the output event-reliability as input, and on the basis of a particular non-linear function $f(\cdot), T_{r}$ is accordingly changed. We do not implement the entire control system, but only a simplified version of it. For instance, we vary $T_{r}$ and observe the behaviour of the system in terms of the mean number of received packets.

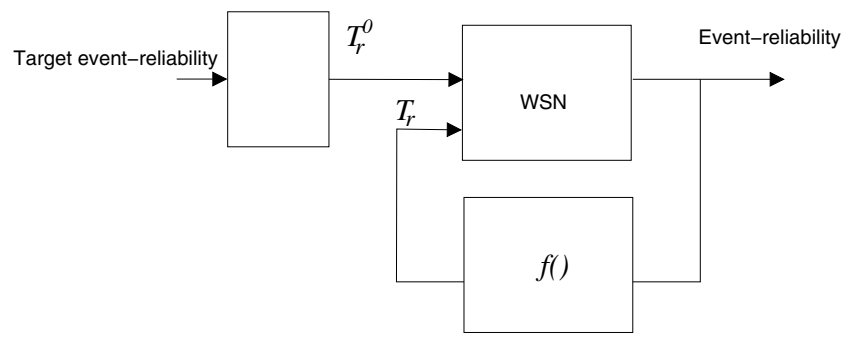

Figure 6. Representation of the transport based on the event-reliability.

In other words, we open the control loop and analyse the forward chain only.

\section{Performance Metrics}

In this paper, we evaluate the performance of the proposed WSNs model by three performance metrics: routing efficiency, delay and number of received packet.

We consider that after a sensor node detects the physical phenomenon, it sends the packets to the sink node via a routing protocol. The ability for transmitting packets for different protocols is different. Also, the RE of a protocol is affected by many network parameters such as wireless transmission radio model, network topology, and transmission frequency [4].

The RE is defined at the sink, and it is the received packet rate divided by the sent packets rate. Thus:

$$
R E(\tau)=\frac{N_{\text {sent }}(\tau)}{N_{\text {routing }}(\tau)}
$$

where $N_{\text {routing }}(\tau)$ is the number of sent packets by routing protocol, and $N_{\text {sent }}(\tau)$ is the number of sent packets by sensor nodes which detect the phenomenon.

To analyse the performance of AODV routing protocol for different radio models, we consider also the delay metric. In this work, we evaluate one-way delay from sensor node to the sink. The delay is the average time for a packet to be transmitted from the sensor nodes to reach the sink.

In order to evaluate the throughput of WSN, we consider also the number of received packet, which is the average number of correct packets received at the sink during $T_{r}$.

\section{Simulation Results}

In this section, we present the simulation results of our proposed WSN. We simulated the network by means of NS2 simulator with the support of NRL libraries. It should be noted that since the number of scheduler events within a simulated WSN can be very high, we applied a patch against the scheduler module of NS-2 in order to speed up the simulation time [15]. 
Table I

TOPOLOGY SETTINGS.

\begin{tabular}{|c|c|}
\hline \multicolumn{2}{|c|}{ Lattice } \\
\hline \hline Step & $d=\frac{L}{\sqrt{N}-1} \mathrm{~m}$ \\
\hline Service Area Size & $L^{2}=(800 \mathrm{x} 800) \mathrm{m}^{2}$ \\
\hline Number of Nodes & $N=16,64,100$ \\
\hline Transmission Range & $r_{0}=d$ \\
\hline \hline \multicolumn{2}{|c|}{ Random } \\
\hline Density(nodes $\left./ \mathrm{m}^{2}\right)$ & $\rho \in\left\{25 \cdot 10^{-6}, 2 \cdot 10^{-4}\right\}$ \\
\hline Transmission Range(m) & $r_{0}=180$ \\
\hline
\end{tabular}

Table II

RADIO MODEL AND SYSTEM PARAMETERS.

\begin{tabular}{|c|c|}
\hline \multicolumn{2}{|c|}{ Radio Model Parameters } \\
\hline \hline Path Loss Coefficient & $\alpha=2.7$ \\
\hline Variance & $\sigma_{\mathrm{dB}}^{2}=16 \mathrm{~dB}$ \\
\hline Carrier Frequency & $916 \mathrm{MHz}$ \\
\hline Antenna & omni \\
\hline Threshold (Sensitivity) & $\gamma=-118 \mathrm{~dB}$ \\
\hline \multicolumn{2}{|c|}{ Other Parameters } \\
\hline \hline Reporting Frequency & $T_{r}=[0.1,1000] \mathrm{pps}^{1}$ \\
\hline Interface Queue Size & 50 packets \\
\hline UDP Packet Size & 100 bytes \\
\hline Detection Interval $\tau$ & $30 \mathrm{~s}$ \\
\hline
\end{tabular}

${ }^{1}$ packet per seconds

In Tables I and II, we present the values of parameters used in our WSN. Let us note that the power values concern the power required to transmit and receive one bit, respectively. They do not refer to the radiated power at all. This is also the energy model implemented in the widely used NS-2 simulator.

In this work, we carried out simulation scenarios in the case of mobile sink considering TwoRayGround and Shadowing radio models, AODV routing protocol, lattice topology and different number of sensor nodes. The average values of RE, delay and number of received packets are computed over 20 simulation runs, and they are plotted from Fig. 7 to Fig. 12.

In Fig. 7 and Fig. 8, are shown the average value of RE in case of mobile sink for TwoRayGround and Shadowing radio models, respectively. The RE is an increasing function of $T_{r}$, because as $T_{r}$ increases, the number of sent packet by sensing node is higher than the number of packets used by routing protocol. It should be noted that when the number of sensor nodes is increased, then the number of routes is increased, thus the searching time to find a route also is increased. When the number of nodes is 100 , the RE is the worst in the simulations.

Comparing the results of Fig. 7 and Fig. 8, we can see that for small number of sensor nodes (16 nodes) the RE of Shadowing radio model is better than TwoRayGround model. However, for high node density (100 nodes), the RE of TwoRayGround model is better than Shadowing radio model. The explanation of this effect is not simple, because it is intermingled with the dynamics of MAC and routing protocol. However, intuitively we can say that RE is affected by the sink mobility and radio models.

The results of delay are shown in Fig. 10 and Fig. 9 for TwoRayGround and Shadowing radio models, respectively. In the simulation results, we can see three zones. When $T_{r}$ is less 10 , the delay is small. When the $T_{r}$ is between 10 and 100 , the delay of both radio models models increased with the increase of $T_{r}$. Also, with the increase of node density, the delay is increased. This is because when the number of nodes is increased, the number of routes is increased, thus the transmission time (delay) increases. For $T_{r}$ more than 100 , the delay start to be saturated, however for high node density, it has many oscillations.

Comparing Fig. 10 and Fig. 9, we can see that when $T_{r}$ is less 10, the delay of Shadowing model is better than TwoRayGround model. For Shadowing model is almost zero. When $T_{r}$ is between 1 and 10, the delay for both radio models increases. However, the performance of TwoRayGround model is slightly better than Shadowing. For $T_{r}$ larger than 100, the delay of TwoRayGround model is better than Shadowing model.

In Fig. 11 and Fig. 12, we show the simulation result for number of received packets for TwoRayGround and Shadowing models, respectively. With increase of the number of nodes, the number of received packets is also increased. For TwoRayGround model, when $T_{r}$ is less than 10 , the number of received packets for 16, 64, and 100 nodes is almost the same. However, comparing with Shadowing model, for the same time interval and for the same number of nodes, the number of received packets is less than TwoRayGround model.

The maximum number of received packets for Shadowing model is about 2500. However, the maximum number of received packets for TwoRayGround model is about 4500 . This is caused by the irregularity of Shadowing model. When $T_{r}$ is increased the packet loss of Shadowing model in increased. However, it should be noted that the values of number of received packets of Shadowing model are more stable than TwoRayGround model.

\section{Conclusions}

In this paper, we presented our simulation results for a WSN in case of mobile sink. For simulations, we considered TwoRayGround and Shadowing radio models, AODV protocol, lattice topology and different number of nodes. We used the RE, delay and number of received packets as metrics to measure the WSN performance.

From the simulation results, we conclude as follows.

- The RE is an increasing function of $T_{r}$. With increase of $T_{r}$, the number of sent packet by sensing node is higher than the number of packets used by routing protocol. It should be noted that when the number of sensor nodes is increased, then the number of routes is increased, thus the searching time to find a route also is increased. 


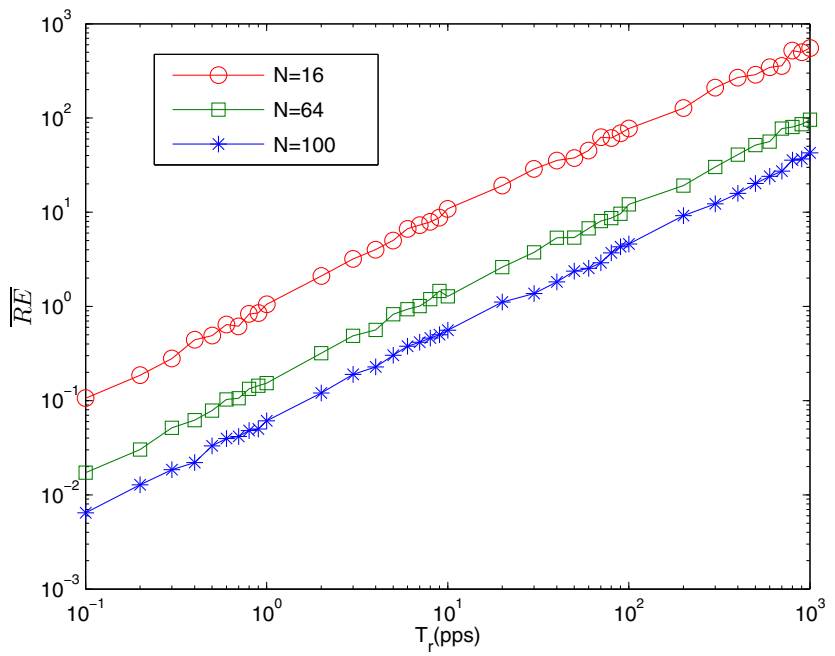

Figure 7. RE for mobile sink with TwoRayGround.

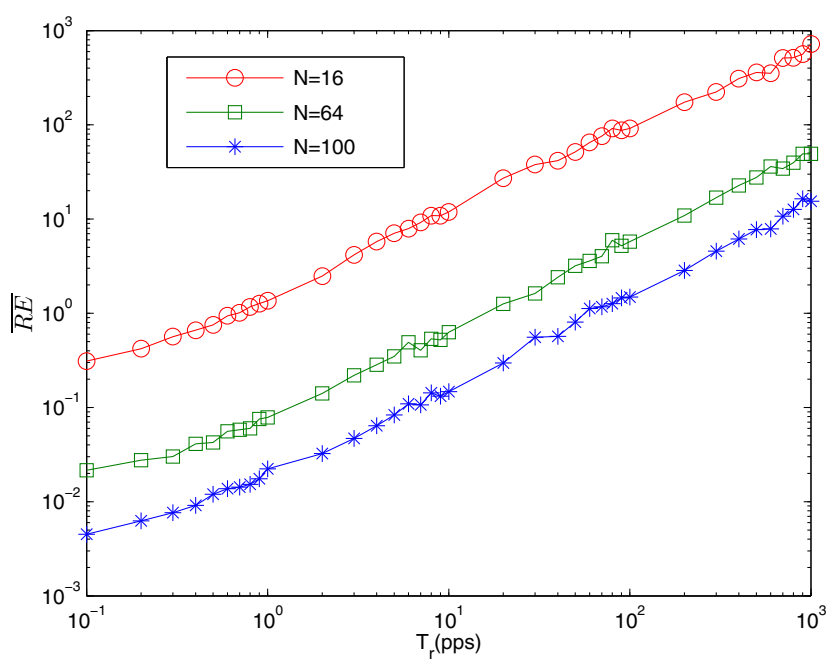

Figure 8. RE for mobile sink with Shadowing.

When the number of nodes is 100 , the RE is the worst in the simulations.

- For small number of sensor nodes (16 nodes), the RE of Shadowing radio model is better than TwoRayGround model. However, for high node density (100 nodes), the $\mathrm{RE}$ of TwoRayGround model is better than Shadowing radio model.

- When $T_{r}$ is less 10 , the delay of Shadowing model is better than TwoRayGround model. When $T_{r}$ is between 1 and 10, the delay for both radio models increases. However, the performance of TwoRayGround model is slightly better than Shadowing. For $T_{r}$ larger than 100 , the delay of TwoRayGround model is better than Shadowing model.

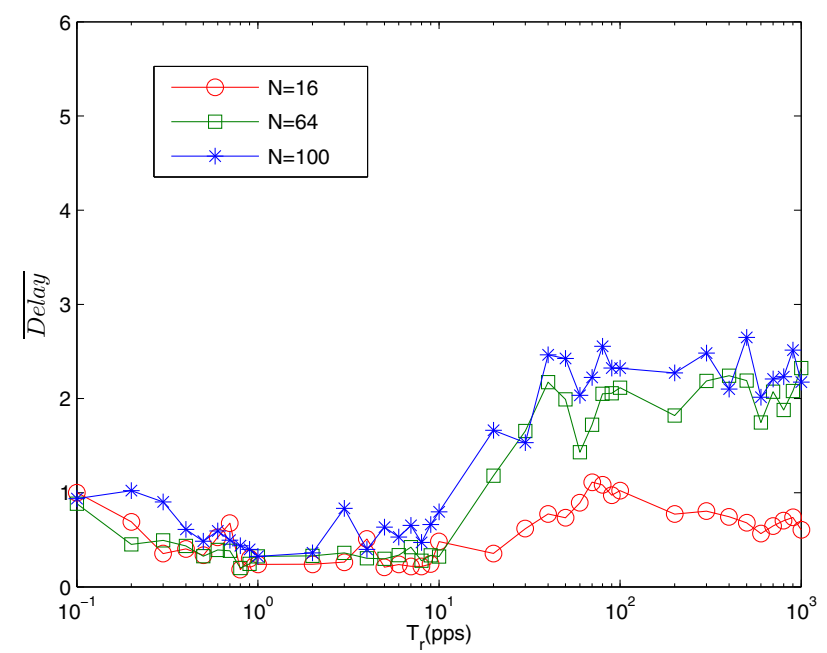

Figure 9. Delay for mobile sink using TwoRayGround.

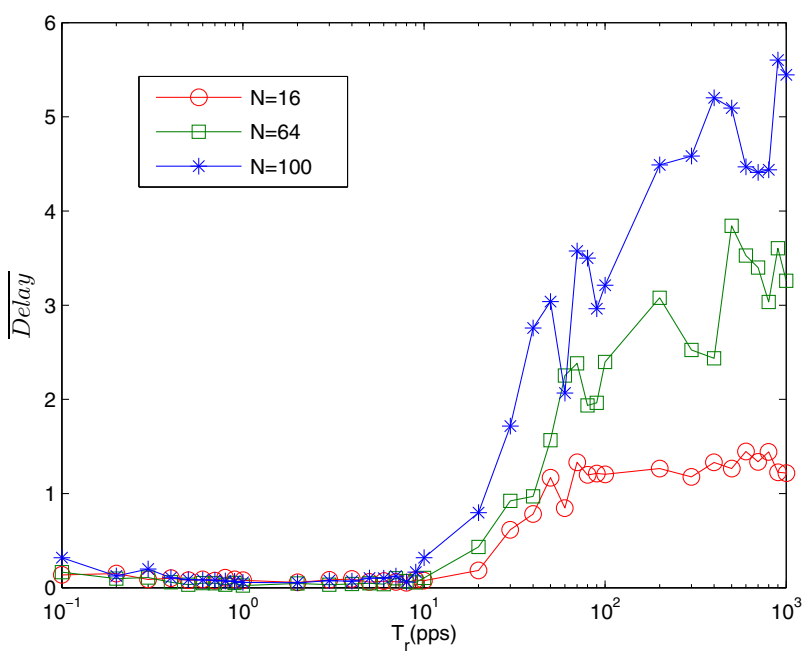

Figure 10. Delay for mobile sink using Shadowing.

- With increase of the number of nodes, the number of received packets is also increased. For TwoRayGround model, when $T_{r}$ is less than 10, the number of received packets for 16, 64, and 100 nodes is almost the same. However, comparing with Shadowing model, for the same time interval and for the same number of nodes, the number of received packets is less than TwoRayGround model.

- The maximum number of received packets for Shadowing model is about 2500. However, the maximum number of received packets for TwoRayGround model is about 4500 . This is caused by the irregularity of Shadowing model. When $T_{r}$ is increased the packet loss of Shadowing model in increased. 


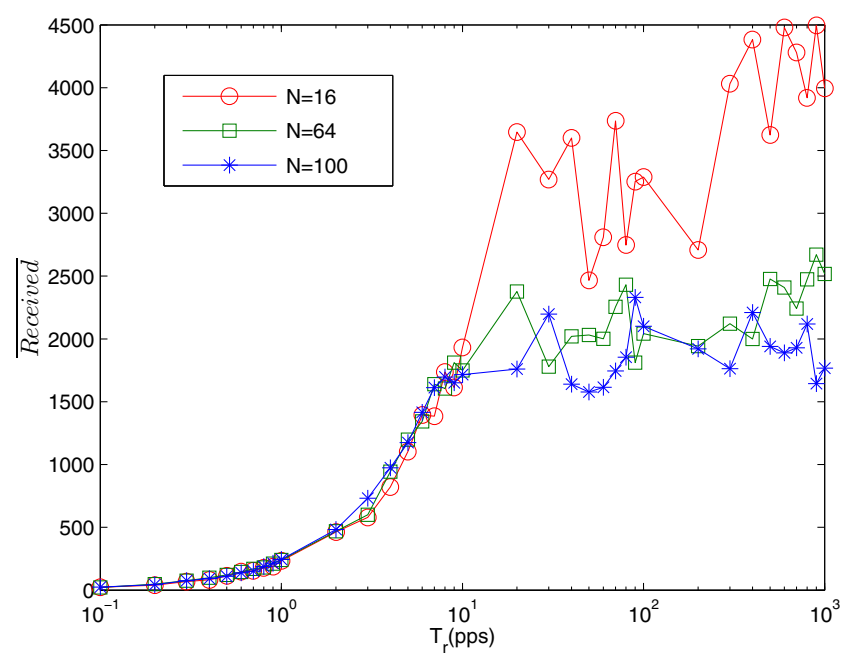

Figure 11. Received packets for mobile sink using TwoRayGround.

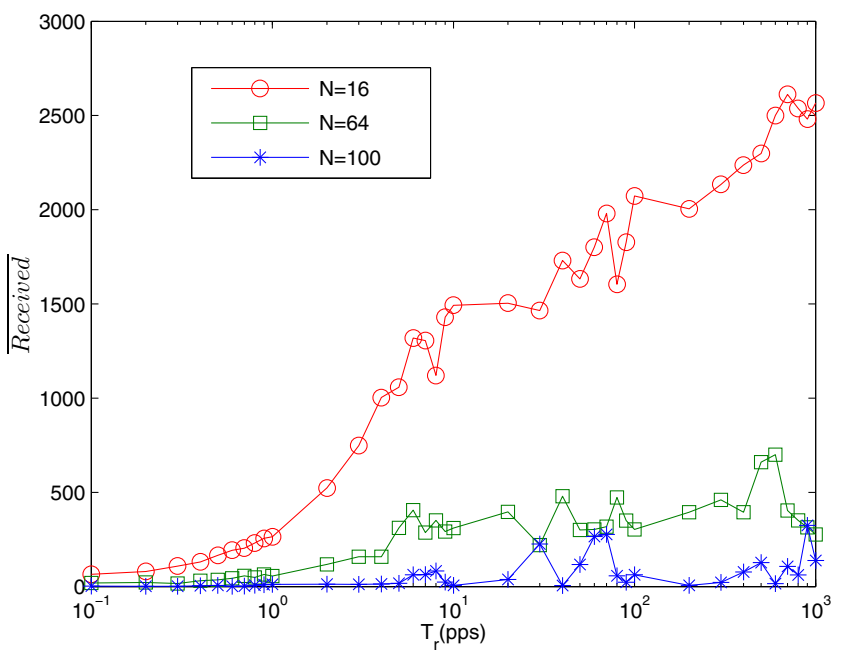

Figure 12. Received packets for mobile sink using Shadowing.

- The values of number of received packets of Shadowing model are more stable than TwoRayGround model.

In the future, we would like to carry out more extensive simulations for multi-mobile sinks. We also would like to consider the case of special movement path for the sink.

\section{ACKNOWLEDGEMENT}

The authors would like to thank KDDI Foundation and Japanese Society for the Promotion of Science (JSPS) for supporting this work.

\section{REFERENCES}

[1] S. Giordano and C. Rosenberg, "Topics in Ad Hoc and Sensor Networks", IEEE Communication Magazine, Vol. 44, No. 4, pp. 97-97, 2006.
[2] W. Hu, N. Bulusu, and S. Jha, "Overload Traffic Management for Sensor Networks", ACM Transactions on Sensor Networks, Vol. 3, No. 4, pp. 18-38, October 2007.

[3] A. A. Somasundara, A. Kansal, D.D. Jea, Jea, D.Estrin, and M. B. Srivastava, "Controllably Mobile Infrastructure for Low Energy Embedded Networks", IEEE Transactions on Mobile Computing, Vol. 5, No. 8, pp. 958-973, August 2006.

[4] O. Younis, S. Fahmy, "HEED: A Hybrid, Energy-efficient, Distributed Clustering Approach for Ad-hoc Sensor Networks", IEEE Transactions on Mobile Computing, Vol. 3, No. 4, pp. 366-379, 2004.

[5] T. Yang, G. De Marco, M. Ikeda, and L. Barolli, "Impact of Radio Randomness on Performances of Lattice Wireless Sensor Networks based on Event-Reliability Concept", International Journal of Mobile Information Systems (MIS), Vol. 2, No. 4, pp. 211-227 2006.

[6] P. Baruah, R. Urgaonkar and B. Krishnamachari, "LearningEnforced Time Domain Routing to Mobile Sinks in Wireless Sensor Fields", Proc. of 29th Annual IEEE International Conference on Local Computer, Tampa, FL, USA, pp. 525532, November 2004.

[7] J. Luo and J.-P. Hubaux, "Joint Mobility and Routing for Lifetime Elongation in Wireless Sensor Networks", Proc. of the 24th The Conference on Computer Communications (Infocom 2005), Miami, FL, USA, pp. 1735-1746, March 2005.

[8] T. Yang, M. Ikeda, G. Mino, L. Barolli, A. Durresi and F. Xhafa, "Performance Evaluation of Wireless Sensor Networks for Mobile Sink Considering Consumed Energy Metric", Proc. of AINA-2010, Perth, Australia, pp. 245-250, April 2010.

[9] I. Donward, "NRL's Sensor Network Extension to NS-2", http://pf.itd.nrl.navy.mil/analysisnrlsensorsim/, 2004.

[10] Crossbow technology, inc. http://www.xbow.com/.

[11] T. S. Rappaport, "Wireless Communications", Prentice Hall PTR, 2001.

[12] Z. Vincze, K. Fodor, R. Vida, A. Vidacs, "Electrostatic Modelling of Multiple Mobile Sinks in Wireless Sensor Networks", Proc. of the IFIP Networking Workshop on Performance Control in Wireless Sensor Networks (PWSN 2006), Coimbra, Portugal, pp. 30-37, May 2006.

[13] C. Perkins, ”Ad Hoc Networks", Addison-Wesley, 2001.

[14] Ö. B. Akan and I. F. Akyildiz "Event-to-Sink Reliable Transport in Wireless Sensor Networks", IEEE/ACM Transactions on Networking, Vol. 13, No. 5, pp. 1003-1016, 2005.

[15] D. X. Wei. Speeding up ns-2 scheduler. http://netlab.caltech.edu/ weixl/technical/ns2patch, September 2005. California Institute of Technology. 